\title{
RESEARCH
}

Open Access

\section{Chemotherapy-induced cognitive impairment in hematological malignancies}

Mohamed Gamal Kotb, Abd El Rahman Soliman, Rasha Ibrahim Ibrahim, Rasha Magdy Mohamed Said and Mona Mokhtar Wahid El Din ${ }^{*}$ (D)

\begin{abstract}
Objectives: Chemotherapy-induced cognitive impairment $(\mathrm{ClCl})$ is one of the most prominent side effects as it negatively impacts activities of daily life of the affected role. These problems can range from subtle to severe and last for months or years after discourse. As cognition is an important predictor of survival in patients with hematological malignancy, savvy factors that lead to $\mathrm{CICl}$ in hematological malignancies warrant attention.

Patients and methods: This is a cross-sectional discipline that was conducted at the Clinical Hematology Section in Ain Shams University Hospital during the period from March 2017 to September 2017. We studied the prevalence of cognitive deterioration among treated patients with chemotherapy for hematological malignancy, and we described its correlation to demographic data and risk agent. Test of cognitive function has been done by using Montreal Cognitive Assessment (MoCA).

Results: Out of 150 patients with different hematological malignancies who finished their chemotherapy at least 6 month ago, we found that 93 patient roles (62\%) are cognitively impaired. The average score of Montreal test for all patient role was $23.913 \pm 3.997$. $\mathrm{ClCl}$ is more among patient who received parenteral chemotherapies and closely related to premedication comorbidities, and all patients with myelodysplastic syndrome (MDS) were cognitively impaired. Also, there was a positive correlation between patient age and cognitive handicap as mean age of patients with abnormal cognitive function was $51.151 \pm 9.933$ ( $p$ value $<0.001$ ) while period of hospital admission was showing significant correlation with impaired abstraction function ( $p$ value 0.003 ), and number of chemotherapy cycles showed significant correlation with naming and orientation cognitive impairment ( $p$ value 0.029 and 0.022 , respectively). We found that female patients had significant shortcoming in naming ingredient more than male ( $p$ value 0.009 ). The type of chemotherapy regimen received did not significantly affected the overall cognitive impairment, but patients who had received Velcade-based chemotherapy had significantly lower executive and abstract function ( $p$ value 0.026). Patient roles which did not achieve remission at follow-up have markedly significant lower scores of most of the cognitive social function.
\end{abstract}

Conclusion and recommendation: $\mathrm{ClCl}$ is a major problem in patient role with hematological malignance postchemotherapy that can affect their quality of life, so fixture follow-up of the cognitive functions in those patients for early interference with proper management of risk factor is recommended.

Keywords: Cognitive impairment, Chemotherapy, Hematological malignancies

\footnotetext{
*Correspondence: Wahid_el_din@hotmail.com

Faculty of Medicine, Ain Shams University, Cairo, Egypt
} 


\section{Introduction}

Hematological malignancies include a diverse radical of lymphomas and leukemia that arise in cells of the immune and lymphatic systems [1]. Improvements in both the diagnosis and treatment of hematological malignancies have markedly increased natural selection for many patients. Based on the recent studies, current 5-year survival rates are as follows: leukemia 60.3\%, Hodgkin's lymphoma (HL) 87.7\%, and non-Hodgkin's lymphoma (NHL) 71. $4 \%$ [1].

Chemotherapy has improved the outcomes for patients with cancer markedly [2]. Nearly all leukemia and 69\% of NHL are treated with chemotherapeutic agents [3]. Chemotherapy has a range of side effects that depend on the case of medications used. Most of chemotherapy medications affect mainly the rapidly dividing cells of the body, such as blood cells and the mucous cells that facing the mouth, stomach, and intestines. Chemotherapy-related toxicities can occur acutely after administration, within hours or days, or chronically, from weeks to year [4].

Cognitive impairment is a very important side effect that can decrease treatment conformity and ultimately impact lineament of life; however, a deep understanding of the etiology of these cognitive job as a consequence of disease and/or treatment among hematological malignancy patients is still not declared [5]. Chemotherapy-induced cognitive impairment (CICI) is an accumulation of job related to memory, attention, concentration, and executive procedure. These problems can range from subtle to severe, and they can last for months or years after treatment [6].

Chemotherapy-induced cognitive impairment affects an estimated 10 million cancer survivor in the USA Based on data from all cases of cancer, up to $30 \%$ of cancer survivors experience cognitive impairment prior to therapy and $80 \%$ during therapy, and up to $35 \%$ may live with CICI up to 20 years after handling [7]. Decreased cognitive function is often associated with poorer quality of life, inability to achieve work and educational goals, inability to drive or read, and decreased social connectedness [8].

The growing lit in this area suggests that cognition is an important forecaster of survival in patients with hematological malignancy and therefore understanding such factors that lead to CICI in hematological malignancies warrants attention [9].

\section{Patients and methods}

This is a cross-sectional discipline that was conducted at the Clinical Hematology Department of Ain Shams University Hospital during the period from March 2017 to September 2017. The study included 150 patients of same socioeconomic status of middle age group (18-55 years) with different hematological malignancy that received different chemotherapy regimens according to the type of malignancies.

All patient roles finished their chemotherapy from 6 months to 2 years ago. Written informed consent was obtained from all participants. Excommunication criteria comprise all other disorder element that could influence cognitive function, e.g., patients who are previously diagnosed with epilepsy, stroke, or any other neurological insults, or those who have story of inhalation psychotropic drugs; patients with end stage renal, hepatic, or respiratory disease; and also patients who received cranial radioactivity or bone marrow transplantation.

We studied the prevalence of CICI among hematological malignancy patients post-chemotherapy, and we described its correlation to demographic data, risk factors, nature of disorder, and chemotherapy that had been received.

All topics underwent all of the following:

- Full history taking and thorough physical examination

- Laboratory work-up including complete blood picture and blood chemistry including liver function tests, kidney function tests, serum electrolytes, and $\mathrm{LDH}$

- Viral markers for HCV, HBV, and HIV

- Bone marrow aspirate, trephine biopsy, and CSF analysis (if indicated)

- Radiological work-up including chest x-ray, CT, and MRI (if indicated)

- Test of cognitive function has been done by using Montreal Cognitive Assessment (MoCA) that takes about $10-15 \mathrm{~min}$.

Montreal Cognitive Assessment includes the following:

- Alternating trial making $(0-1)$

- Visuoconstructional skills (cylinder) (0-1)

- Visuoconstructional skills (clock) (0-3)

- Naming (0-3)

- Memory

- Attention(0-2)

- Vigilance (0-1)

- Serial 7s (0-3)

- Sentence repetition (0-2)

- Verbal fluency (0-1)

- Abstraction $(0-2)$

- Delayed recall (0-5)

- Orientation (0-6)

- 12 years or more of formal education (got extra 1 point)

The total score is the sum of all above consecutive tests scores of 30 point. A final total score of less than 26 is considered subnormal. 
Montreal cognitive test was selected to assess mild cognitive impairment and some of the executive functions; MoCA test is superior in MCI detection and is used for screening in VCI (Conti et al, 2015). MoCA's design is superior in sensitivity for detecting MCI. It involves more words, fewer learning trials, and a longer delay before recall than other tests as MMSE. It measures executive functions, higher level language abilities, and complex visuospatial processing with numerous and demanding tasks. The MoCA has more emphasis on tasks of frontal executive functions and attention, making it more sensitive in detecting non-AD dementia.

The Arabic MoCA version is identical to the English version. It was validated in reference to CAMCOG, by Abdel Rahman and El Gafaary in 2009 [10].

\section{Statistical analysis}

Data were collected, revised, coded, and entered to Statistical Package for Social Science (SPSS) version 20.

\section{Results}

Our study included 150 patients, $64.67 \%$ of them were males (97) and $35.33 \%$ were females (53), and their age ranged from 18 to 55 with a mean of $45.533 \pm 12.877$.
The demographic and clinical characteristics of all participants are shown in (Table 1). Our AML patients received 3 days daunarubicin plus 7 days cytarabin followed by HiDAC (high-dose cytarabin) as consolidation, and old age patients received low-dose ara-c. ALL patients received hyper-CVAD protocol, CML patients received one of the TKIs (imatinibe, dasatinibe, or nilotinib), MDS patients received 5-azacitidine, MM patients received velcade and dexamethasone, and NHL patients received rituximab plus cyclophosphamide, vincristine, doxorubicin, and prednisone $(\mathrm{R}+\mathrm{CHOP})$ or cyclophosphamide, vincristine, and prednisone (CVP) while patients with Hodgkin's disease received doxorubicin, bleomycin, vinblastine, and dacarbazine (ABVD), and CLL patients received fludarabine, cyclophosphamide, and rituximab (FCR) or chlorambucil-based therapy.

Mean number of cycles that our patient had received was $4.020 \pm 2.370$, and average day of hospital stay was $33.040 \pm 26.72433$ days.

By using the Montreal test assessment, we found that 93 patients (62\%) are cognitively impaired, while $57 \mathrm{pa}$ tients (38\%) had normal cognitive functions. The average score of Montreal test for all patients was $23.913 \pm 3.997$ with the mean individual test scores as follows: executive was 3.5 of 5 , naming was 2.96 of 3 , attention was 3.7 of 5 ,

Table 1 Demographic and clinical characteristics of all participants of the study

\begin{tabular}{|c|c|c|c|}
\hline & & Number & Percentage \\
\hline \multirow[t]{7}{*}{ Diagnosis } & AML & 45 & 30.00 \\
\hline & ALL & 15 & 10.00 \\
\hline & CML & 16 & 10.67 \\
\hline & CLL & 20 & 13.33 \\
\hline & MDS & 2 & 1.33 \\
\hline & Lymphoma & 25 & 16.67 \\
\hline & MM & 27 & 18.00 \\
\hline \multirow[t]{4}{*}{ Chemotherapy protocol } & Intensified protocol & 56 & 37.33 \\
\hline & TKls & 13 & 8.67 \\
\hline & Velcade & 27 & 18.00 \\
\hline & Others & 54 & 36.00 \\
\hline \multirow[t]{2}{*}{ Route of administration chemotherapy } & Oral & 15 & 10.00 \\
\hline & Parenteral & 135 & 90.00 \\
\hline \multirow[t]{5}{*}{ Comorbidities } & No comorbidities & 104 & 69.33 \\
\hline & HTN & 16 & 10.67 \\
\hline & DM & 10 & 6.67 \\
\hline & Viral hepatic infection & 8 & 5.33 \\
\hline & More than one & 12 & 8.00 \\
\hline \multirow[t]{2}{*}{ Remission status } & Remitted & 103 & 68.67 \\
\hline & Resistant or refractory & 47 & 31.33 \\
\hline
\end{tabular}

AML acute myeloid leukemia, ALL acute lymphoblastic leukemia, CML chronic myeloid leukemia, CLL chronic lymphocytic leukemia, MDS myelodysplastic syndrome, MM multiple myeloma, TKIs tyrosine kinase inhibitors, HTN hypertension, DM diabetes mellitus. Intensified protocols include the following: $3+7$, HIDAC hyper-CVAD, FCR, ABVD, and R-CHOP. Other chemotherapies include the following: low-dose ara-c, 5-azacitidine, CVP, and chlorambucil-based therapy 
language was 2.57 of 3 , sbstraction was 1.08 of 2 , delayed recall was 4.2 of 5 , and orientation was 5.8 of 6 .

The comparison between group of patients with normal overall Montreal score and those patients with abnormal score (Table 2) revealed that patients who received parenteral chemotherapy had significantly abnormal Montreal score more than those who received oral chemotherapy ( $p$ value 0.016$)$, and also, patients with MDS had the lowest Montreal score ( $p$ value 0.021 ); moreover, there is a highly significant relation between cognitive impairment and comorbidities ( $p$ value $<0.001$ ).

There was a positive correlation between patient age and cognitive impairment as the mean age of patients with normal cognitive function was $36.368 \pm 11.883$ versus patients with abnormal cognitive function $51.151 \pm$ 9.933 ( $p$ value $<0.001)$; moreover, age is significantly correlated with defect in all components of cognitive function ( $p$ value $<0.001$ ) except naming and orientation (Table 3).

Period of hospital admission was showing no significant correlation with the overall cognitive impairment except with impairment in abstraction function ( $p$ value 0.003), while the number of chemotherapy cycles which had been received by patients is showing a significant correlation with naming and orientation cognitive impairment ( $p$ value 0.029 and 0.022 , respectively).

Both Tables 4 and 5 showed impact of some variables on different cognitive functions. We found that female patients had significant defect in naming component more than male ( $p$ value 0.009). Patients who received parenteral chemotherapy had significant affection in executive and abstraction function more than those who received oral chemotherapy ( $p$ value $<0.001$ and 0.020 , respectively).

The chemotherapy regimen received did not significantly affected the overall cognitive impairment. But patients who received Velcade-based chemotherapy had significantly lower executive and abstraction function ( $p$ value 0.026 ). Affected roles which did not achieve remission at follow-up have markedly significant lower scores of different cognitive functions ( $p$ value 0.001 ) except abstraction and attention functions.

\section{Discussion}

Chemotherapy (CT) is the cornerstone therapy for almost all hematological malignancies. Advances in CT protocols with better supportive care and earlier

Table 2 Comparison between group of patients with normal overall Montreal score and those patients with abnormal score

\begin{tabular}{|c|c|c|c|c|c|c|c|}
\hline & & \multicolumn{4}{|c|}{ Overall Montreal score } & \multirow{2}{*}{\multicolumn{2}{|c|}{ Chi-square }} \\
\hline & & \multicolumn{2}{|c|}{ Normal } & \multicolumn{2}{|c|}{ Cognitive impairment } & & \\
\hline & & $\bar{N}$ & $\%$ & $\bar{N}$ & $\%$ & $x^{2}$ & $p$ value \\
\hline \multirow[t]{2}{*}{ Sex } & Male & 40 & 70.18 & 57 & 61.29 & 1.22 & 0.26 \\
\hline & Female & 17 & 29.82 & 36 & 38.71 & & \\
\hline \multirow[t]{7}{*}{ Diagnosis } & AML & 17 & 29.82 & 28 & 30.11 & 14.91 & 0.02 \\
\hline & ALL & 8 & 14.04 & 7 & 7.53 & & \\
\hline & $C M L$ & 11 & 19.30 & 5 & 5.38 & & \\
\hline & $C L L$ & 6 & 10.53 & 14 & 15.05 & & \\
\hline & MDS & 0 & 0.00 & 2 & 2.15 & & \\
\hline & Lymphoma & 4 & 7.02 & 21 & 22.58 & & \\
\hline & Myeloma & 11 & 19.30 & 16 & 17.20 & & \\
\hline \multirow[t]{4}{*}{ Chemotherapy } & Intensified protocol & 23 & 40.35 & 33 & 35.48 & 5.76 & 0.12 \\
\hline & TKls & 8 & 14.04 & 5 & 5.38 & & \\
\hline & Velcade & 11 & 19.30 & 16 & 17.20 & & \\
\hline & Others & 15 & 26.32 & 39 & 41.94 & & \\
\hline \multirow[t]{2}{*}{ Route of chemotherapy } & Oral & 10 & 17.54 & 5 & 5.38 & 5.81 & 0.01 \\
\hline & Parenteral & 47 & 82.46 & 88 & 94.62 & & \\
\hline \multirow[t]{5}{*}{ Comorbidities } & No & 53 & 92.98 & 51 & 54.84 & 26.52 & $<0.001$ \\
\hline & HTN & 0 & 0.00 & 16 & 17.20 & & \\
\hline & DM & 2 & 3.51 & 8 & 8.60 & & \\
\hline & Viral hepatic infection & 2 & 3.51 & 6 & 6.45 & & \\
\hline & More than one & 0 & 0.00 & 12 & 12.90 & & \\
\hline \multirow[t]{2}{*}{ Remission status } & Remitted & 42 & 73.68 & 61 & 65.59 & 1.07 & 0.30 \\
\hline & Resistant or refractory & 15 & 26.32 & 32 & 34.41 & & \\
\hline
\end{tabular}


Table 3 The correlation of the age of patients, number of chemotherapy cycles received, and period of hospital admission with each component of Montreal scoring system

\begin{tabular}{|c|c|c|c|c|c|c|}
\hline & \multicolumn{2}{|l|}{ Age } & \multicolumn{2}{|c|}{ No. of cycles } & \multicolumn{2}{|c|}{ Hospital stay (days) } \\
\hline & $r$ & $p$ value & $r$ & $p$ value & $r$ & $p$ value \\
\hline Executive & -0.44 & $<0.001$ & 0.07 & 0.37 & 0.01 & 0.84 \\
\hline Naming & 0.02 & 0.97 & -0.17 & 0.02 & -0.07 & 0.37 \\
\hline Attention & -0.48 & $<0.001$ & 0.07 & 0.36 & 0.06 & 0.43 \\
\hline Language & -0.31 & $<0.001$ & 0.08 & 0.30 & -0.18 & 0.11 \\
\hline Abstraction & -0.34 & $<0.001$ & 0.11 & 0.16 & -0.24 & 0.003 \\
\hline Delayed recall & -0.41 & $<0.001$ & -0.03 & 0.67 & -0.16 & 0.04 \\
\hline Orientation & -0.15 & 0.06 & -0.18 & 0.02 & -0.10 & 0.21 \\
\hline Total score & -0.44 & $<0.001$ & 0.09 & 0.25 & -0.10 & 0.22 \\
\hline
\end{tabular}

detection of its toxicity will lead to an increase in the number of patients undergoing complete remission with the least toxicity [10].

However, the chemotherapy intake is often accompanied by many side effects involving immunosuppression, myelosuppression, gastrointestinal distress, anemia, hair loss, secondary neoplasm, infertility, teratogenicity, peripheral neuropathy, cognitive impairment, tumor lysis syndrome, and organ damage [4].

Some cognitive problems in those who receive chemotherapy are more severe than in those who only receive locoregional therapy (e.g., radiation, surgery), and it can negatively impact activities of daily living $[8,11]$.

Most of the previous studies were targeting the correlation between chemotherapy protocol intake and the cognitive functions without considering the other variable factors. Also, few of the previous studies concerning with CICI were targeting hematological malignancies in specific. That is why we had targeted our study for the discussion of the correlation between cognitive impairment following chemotherapy intake among hematological malignancy patients with multiple factors including the nature of disease, type of chemotherapy regimen, number of its cycles, duration of hospital stay, age of the patient, sex, route of administration of the chemotherapy, remission status of patients, and other comorbidities (hypertension, diabetes, and viral hepatic infections).

In our study, 93 patients (62\%) developed various degrees of cognitive impairment, and 57 patients (38\%) had proven to have normal cognitive functions; this is in accordance with literature which suggests that chemotherapy has a negative impact on cognitive functioning $[12,13]$. Some potential mechanisms of CICI include the following: direct neurotoxic injury to neurons, white or gray matter microvasculature that causes direct ischemia, altered levels of neurotransmitters or DNA damage, and subsequent oxidative stress [5].

Although several positive studies have found evidence supporting the influence of chemotherapy on cognitive functioning [1, 14-17], yet other negative studies have also been reported [18-20]; this discrepancy may be due to one or more of other variable factors as reported by Vardy et al. This included the different chemotherapy regimens administered, variations in normative data and reference groups, difference in tools, and scales used for cognitive impairment diagnosis [21]. Moreover, Peckham reported

Table 4 Impact of patient sex, route of chemotherapy, associated comorbidities, and remission status on the score of each component of cognitive functions

\begin{tabular}{|c|c|c|c|c|c|c|c|c|c|}
\hline \multicolumn{2}{|l|}{ Variables } & \multirow{2}{*}{$\frac{\text { Executive }}{3.54 \pm 0.99}$} & \multirow{2}{*}{$\frac{\text { Naming }}{3.00 \pm 0.00}$} & \multirow{2}{*}{$\frac{\text { Attention }}{3.77 \pm 0.90}$} & \multirow{2}{*}{$\frac{\text { Language }}{2.61 \pm 0.63}$} & \multirow{2}{*}{$\frac{\text { Abstraction }}{1.08 \pm 0.51}$} & \multirow{2}{*}{$\frac{\text { Delayed recall }}{4.28 \pm 0.95}$} & \multirow{2}{*}{$\begin{array}{l}\text { Orientation } \\
5.85 \pm 0.47\end{array}$} & \multirow{2}{*}{$\frac{\text { Total score }}{24.16 \pm 3.70}$} \\
\hline Sex & Male & & & & & & & & \\
\hline & female & $3.52 \pm 0.97$ & $2.88 \pm 0.42$ & $3.62 \pm 0.68$ & $2.49 \pm 0.75$ & $1.07 \pm 0.64$ & $4.09 \pm 1.16$ & $5.75 \pm 0.70$ & $23.45 \pm 4.47$ \\
\hline \multirow[t]{2}{*}{$t$ test } & $t$ & 0.10 & 2.64 & 1.05 & 1.10 & 0.07 & 1.09 & 1.04 & 1.04 \\
\hline & $p$ value & 0.91 & 0.009 & 0.29 & 0.27 & 0.94 & 0.27 & 0.30 & 0.29 \\
\hline \multirow[t]{2}{*}{ Route of CT } & Parenteral & $3.44 \pm 0.95$ & $2.95 \pm 0.27$ & $3.69 \pm 0.84$ & $2.54 \pm 0.69$ & $1.04 \pm 0.55$ & $4.17 \pm 1.06$ & $5.80 \pm 0.59$ & $23.65 \pm 4.07$ \\
\hline & Oral & $4.40 \pm 0.50$ & $3.00 \pm 0.00$ & $3.93 \pm 0.79$ & $2.86 \pm 0.35$ & $1.40 \pm 0.50$ & $4.66 \pm 0.48$ & $6.00 \pm 0.00$ & $26.26 \pm 2.08$ \\
\hline \multirow[t]{2}{*}{$t$ test } & $t$ & 3.73 & 0.63 & 1.04 & 1.77 & 2.36 & 1.77 & 1.29 & 2.44 \\
\hline & $p$ value & $<0.001$ & 0.52 & 0.29 & 0.07 & 0.02 & 0.07 & 0.19 & 0.01 \\
\hline \multirow[t]{2}{*}{ Comorbidities } & Yes & $3.13 \pm 0.83$ & $2.95 \pm 0.20$ & $3.39 \pm 0.80$ & $2.32 \pm 0.66$ & $0.80 \pm 0.50$ & $3.73 \pm 1.06$ & $5.69 \pm 0.66$ & $22.04 \pm 3.81$ \\
\hline & No & $3.72 \pm 0.99$ & $2.96 \pm 0.27$ & $3.86 \pm 0.81$ & $2.68 \pm 0.65$ & $1.20 \pm 0.54$ & $4.43 \pm 0.95$ & $5.87 \pm 0.51$ & $24.74 \pm 3.80$ \\
\hline \multirow[t]{2}{*}{$t$ test } & $t$ & 3.53 & 0.11 & 3.30 & 3.04 & 4.21 & 3.96 & 1.79 & 3.99 \\
\hline & $p$ value & 0.001 & 0.91 & 0.001 & 0.003 & $<0.001$ & $<0.001$ & 0.07 & $<0.001$ \\
\hline \multirow[t]{2}{*}{ Remission status } & Remission & $3.66 \pm 0.91$ & $3.00 \pm 0.00$ & $3.81 \pm 0.75$ & $2.68 \pm 0.58$ & $1.10 \pm 0.39$ & $4.42 \pm 0.84$ & $5.92 \pm 0.36$ & $24.61 \pm 2.97$ \\
\hline & Not & $3.27 \pm 1.07$ & $2.87 \pm 0.44$ & $3.51 \pm 0.97$ & $2.34 \pm 0.81$ & $1.02 \pm 0.82$ & $3.76 \pm 1.25$ & $5.59 \pm 0.82$ & $22.38 \pm 5.35$ \\
\hline \multirow[t]{2}{*}{$t$ test } & $t$ & 2.25 & 2.90 & 2.09 & 2.90 & 0.86 & 3.78 & 3.37 & 3.26 \\
\hline & $p$ value & 0.02 & 0.004 & 0.03 & 0.004 & 0.38 & $<0.001$ & 0.001 & 0.001 \\
\hline
\end{tabular}


Table 5 Impact of patient diagnosis and types of chemotherapy on the score of each component of cognitive functions

\begin{tabular}{|c|c|c|c|c|c|c|c|c|c|}
\hline \multicolumn{2}{|l|}{ Variables } & \multirow{2}{*}{$\frac{\text { Executive }}{3.42 \pm 1.07}$} & \multirow{2}{*}{$\frac{\text { Naming }}{2.87 \pm 0.45}$} & \multirow{2}{*}{$\frac{\text { Attention }}{3.55 \pm 0.89}$} & \multirow{2}{*}{$\frac{\text { Language }}{2.42 \pm 0.83}$} & \multirow{2}{*}{$\frac{\text { Abstraction }}{0.97 \pm 0.65}$} & \multirow{2}{*}{$\frac{\text { Delayed recall }}{4.06 \pm 1.26}$} & \multirow{2}{*}{$\frac{\text { Orientation }}{5.64 \pm 0.83}$} & \multirow{2}{*}{$\frac{\text { Total score }}{22.95 \pm 5.21}$} \\
\hline Diagnosis & AML & & & & & & & & \\
\hline & ALL & $3.93 \pm 0.59$ & $3.00 \pm 0.00$ & $4.20 \pm 0.94$ & $2.66 \pm 0.48$ & $0.93 \pm 0.59$ & $4.40 \pm 1.24$ & $5.80 \pm 4.14$ & $24.93 \pm 3.77$ \\
\hline & CML & $4.43 \pm 0.51$ & $3.00 \pm 0.00$ & $3.81 \pm 0.65$ & $2.87 \pm 0.34$ & $1.37 \pm 0.50$ & $4.68 \pm 0.47$ & $6.00 \pm 0.00$ & $26.18 \pm 1.94$ \\
\hline & $C L L$ & $3.45 \pm 0.88$ & $3.00 \pm 0.00$ & $3.80 \pm 1.05$ & $2.70 \pm 0.73$ & $1.05 \pm 0.60$ & $4.00 \pm 1.07$ & $5.70 \pm 0.73$ & $23.70 \pm 4.53$ \\
\hline & MDS & $2.00 \pm 0.00$ & $3.00 \pm 0.00$ & $3.00 \pm 0.00$ & $2.00 \pm 0.00$ & $0.00 \pm 0.00$ & $4.00 \pm 0.00$ & $6.00 \pm 0.00$ & $20.00 \pm 0.00$ \\
\hline & Lymphoma & $3.32 \pm 0.94$ & $3.00 \pm 0.00$ & $3.92 \pm 0.64$ & $2.68 \pm 0.47$ & $1.20 \pm 0.40$ & $4.48 \pm 0.51$ & $6.00 \pm 0.00$ & $24.60 \pm 1.84$ \\
\hline & MM & $3.37 \pm 0.96$ & $3.00 \pm 0.00$ & $3.48 \pm 0.64$ & $2.44 \pm 0.69$ & $1.14 \pm 0.36$ & $4.03 \pm 1.01$ & $5.92 \pm 0.26$ & $23.40 \pm 3.19$ \\
\hline \multirow[t]{2}{*}{ ANOVA } & $F$ & 4.49 & 1.45 & 2.11 & 1.60 & 2.84 & 1.37 & 1.79 & 2.06 \\
\hline & $p$ value & $<0.001$ & 0.19 & 0.05 & 0.15 & 0.01 & 0.23 & 0.10 & 0.06 \\
\hline \multirow[t]{4}{*}{ CT protocol } & Intensified CT & $3.51 \pm 0.99$ & $2.89 \pm 0.41$ & $3.69 \pm 0.93$ & $2.48 \pm 0.78$ & $0.96 \pm 0.66$ & $4.16 \pm 1.27$ & $5.66 \pm 0.76$ & $23.37 \pm 5.02$ \\
\hline & TKIs & $4.30 \pm 0.48$ & $3.00 \pm 0.00$ & $3.76 \pm 0.72$ & $2.84 \pm 0.37$ & $1.46 \pm 0.51$ & $4.61 \pm 0.50$ & $6.00 \pm 0.00$ & $26.00 \pm 2.12$ \\
\hline & Velcade based & $3.37 \pm 0.96$ & $3.00 \pm 0.00$ & $3.48 \pm 0.64$ & $2.44 \pm 0.69$ & $1.14 \pm 0.36$ & $4.03 \pm 1.01$ & $5.92 \pm 0.26$ & $23.40 \pm 3.19$ \\
\hline & Others & $3.46 \pm 1.00$ & $3.00 \pm 0.00$ & $3.85 \pm 0.83$ & $2.66 \pm 0.58$ & $1.07 \pm 0.50$ & $4.27 \pm 0.83$ & $5.88 \pm 0.46$ & $24.22 \pm 3.33$ \\
\hline \multirow[t]{2}{*}{ ANOVA } & $F$ & 3.18 & 2.09 & 1.21 & 1.72 & 3.04 & 1.03 & 2.55 & 1.80 \\
\hline & $p$ value & 0.02 & 0.10 & 0.30 & 0.16 & 0.03 & 0.38 & 0.05 & 0.15 \\
\hline
\end{tabular}

that cognitive dysfunction due to chemotherapy may not appear immediately after treatment but needs at least from 2 to 3 years after treatment cessation [22, 23].

In this study, we assessed the impact of age on the cognition functions and we found a statistically significant impact of aging on the cognitive functions ( $p$ value $<.001)$ that severely affect all cognitive components except naming and orientation. This is in agreement with Loh et al. who reported that advancing age is a known risk factor for cognitive impairment; the investigations of the biological mechanics of CICI in older patient are more complex than in younger patients for several reasons: (1) many mechanisms that are involved in CICI are also involved in comorbid precondition, (2) comorbid conditions can increase vulnerability to CICI by biological mechanisms similar or dissimilar from the mechanisms causing the condition itself, and (3) the use of multiple medications in elderly augments the great undesired CICI [24].

Concerning the effect of premedication comorbidities like hypertension, diabetes, and viral hepatic infections, our study results had shown a statistically significant impact of comorbidities on the cognitive functions ( $p$ value $<0.001$ ), with a greater affection on abstraction and delayed recall. This is in agreement with other previous studies, which considered comorbidity's impact on cognitive functioning irrespective of the cancer diagnosis [25]. Also, Panza et al. is coming in agreement with our results [26].

So careful measurement and assessment of comorbid conditions are particularly important to consider in pretreatment assessment in cancer patients-as the same cytokines and cognate receptors implicated in these diseases are also implicated in CICI [27].
As regards the sex of the patient, our study result did not find an appreciable impact of sex on neurocognitive ability; however, female patients had statistically significant impairment in naming function than male ( $p$ value 0.009). Also, some studies in pediatric group found that girls perform more poorly than boys in assessment of cognitive function tests [28]; this may be explained by sex-based differences in white matter development [29]. On the other hand, males tend to perform better in visuospatial and motor tasks, whereas females tend to perform better in certain verbal areas [30].

In our study, we highlighted the impact of each type of hematological malignancy (ALL, AML, CML, CLL, myeloma, lymphoma, and MDS) among chemotherapeutic treated patients on the cognitive functions. We found that there is a statistically significant impact of type of the malignancy on the cognitive functions ( $p$ value 0.21 ), as all patients with MDS suffered from cognitive impairment, especially executive functions ( $p$ value $<0.001$ ), followed by the abstraction ( $p$ value 0.012 ). Our result in accordance with one cogitation of patient role with MDS or AML patient reported increased cognitive deficits prior to any handling and more dysfunction up to 1 month after therapy; this may be related to the point of circulating cytokine [31]. Another study of patients with MDS and CML reported high pace of impairment at baseline and improvement up to 18 months after treatment [32]. We recommend more subject areas with larger number of patients of each disease for accurate judgment.

In our study, we found that the number of chemotherapy cycles that had been received by patients had statistically significant impact on naming and orientation cognitive function ( $p$ value 0.029 and 0.022 , 
respectively), patients who received Velcade chemotherapy had statistically significant lower executive and abstraction function.

This is in accordance with Jones et al. who found that many myeloma patients are likely to suffer impaired cognitive function after their initial multiple myeloma therapy. The patients had received a median of 3 to 4 cycles of induction therapy with Velcade-based induction therapy, and stem cell transplantation often causes further impairment. The most common signs of cognitive impairment were problems with learning, memory, and coordination [33].

The period of hospital stay is another point which had been discussed in our study, and we found that there is no statistically significant difference between it and the CICI except on abstraction dysfunction ( $p$ value 0.003 ). In contrast, Loh et al. found that long-term hospitalization may increase and worsen the cognitive impairment as it affects the quality of life of the patients in general. Thus, much more studies with longer period of follow-up are needed [34].

When comparing between the effect of oral and parenteral routes of chemotherapy on cognition functions, we found a significant difference being more with parenteral route ( $p$ value 0.16$)$ with highly significant defect in executive part $(p$ value $<0.001)$. However, most studies stress that drugs that can cross BBB (e.g., methotrexate or 5-fluorouracil) has more detrimental effects on cognition function. It was suggested that cytokines play a dominant role in the neuro-immuno-endocrine processes induced by cancer cells and cytotoxic chemotherapy. Chemotherapeutic agents, which are mostly unable to hybridize the blood-brain barrier (BBB) due to their molecular size, can cause toxicity to the brain indirectly via the pro-inflammatory cytokine pathways. It also was suggested that the accumulation of a combination of drug, rather than some drugs, may enhance the neurotoxicity in cancer patient.

Another point of study was the follow-up assessment after 6 months, and its impingement on chemotherapy-induced cognitive handicap revealed no statistically significant divergence. However, some other studies uncovered that deteriorated cognitive affair in children with cancer could be attributed either to cancer treatments including chemotherapy or to cancer per se as some patients who did not achieve remission had poorer cognitive function [35]; this controversy may be explained by relatively short stop of the study and in need for wide scale of patients. So, further larger, prospective, long-term studies are necessary to definitively assess this impairment.

To summarize, there is a necessary need of more comprehensive neuropsychological tests for specific information about the nature and severity of intellectual dysfunction at different times throughout the distance of the discourse full point and even for long terminus survivors as a second step to provide an improved scene for specific effect related to cognitive dysfunction in patients with hematological malignancies. Besides, studies on big sample distribution are also advised to clarify the role of predisposing variables.

Furthermore, the growth of interference that addresses cognitive late answer is imperative, and intervention studies can be more successfully created to target the actual mechanisms to try and prevent cognitive impairment from occurring and address it once it has happened.

\begin{abstract}
Abbreviations
ALL: Acute lymphocytic leukemia; AML: Acute myeloid leukemia; BBB: Bloodbrain barrier; $\mathrm{ClCl}$ : Chemotherapy-induced cognitive impairment;

CLL: Chronic lymphocytic leukemia; CML: Chronic myeloid leukemia; HBV: Hepatitis B virus; HCV: Hepatitis C Virus; HiDAC: High-dose cytarabine; HIV: Human immunodeficiency virus; HL: Hodgkin's lymphoma; LDH: Lactate dehydrogenase; MDS: Myelodysplastic syndrome; MoCA: Montreal Cognitive Assessment; NHL: Non-Hodgkin's lymphoma; SPSS: Statistical Package for Social Science; TKIs: Tyrosine kinase inhibitors
\end{abstract}

\section{Acknowledgements}

Not applicable.

Authors' contributions

MK carried out the cognitive tests, patient selection, and statistical analysis. RM and RI selected the project, designed the study, determined the criteria of the patients, participated in the sequence alignment, drafted the manuscript, and revised the statistical tests and analysis. AS conceived of the study and participated in its design and coordination. All authors read and approved the final manuscript.

\section{Funding}

Self-financing.

\section{Availability of data and materials}

All data and materials that support the findings in this manuscript are available. Please contact the authors for data requests.

\section{Ethics approval and consent to participate}

The procedures followed were in accordance with the ethical standards of the responsible committee on human experimentation and with the principles of Helsinki Declaration. Informed consent was obtained from all participants, and ethical approval from the Neuropsychiatry Department Ethical Committee of Ain Shams University was obtained on 20 April 2017 before starting our work.

Consent for publication

Not applicable.

\section{Competing interests}

The authors declare that they have no competing interests.

Received: 28 July 2018 Accepted: 9 August 2019

Published online: 22 August 2019

\section{References}

1. Howlader N, Noone AM, Krapcho M, et al. (2014): SEER cancer statistics review, 1975-2011, National Cancer Institute.

2. VT DV, Lawrence TS, Rosenberg SA. Cancer: principles and practice of oncology. 10th ed. Philadelphia (PA): Lippincott Williams \& Wilkins; 2015. p. $1526-51$.

3. Rossi D, Terzi-di-Bergamo L, De Paoli L, et al. Molecular prediction of durable remission after first-line fludarabine-cyclophosphamide-rituximab in chronic lymphocytic leukemia. Blood. 2015;126:1921-4.

4. MacDonald V. Chemotherapy: managing side effects and safe handling. The Canadian Veterinary Journal. 2009;50(6):665-8.

5. Wefel JS, Lenzi R, Theriault RL, Davis RN, Meyers CA. The cognitive sequelae of standard-dose adjuvant chemotherapy in women with breast carcinoma. 
Results of a prospective, randomized, longitudinal trail. Cancer. 2004;100(11): 2292-9.

6. Bradley CJ, Neumark D, Bednarek HL, Schenk M. Short-term effects of breast cancer on labor market attachment: results from a longitudinal study. Journal of Health Economics. 2005;24:137-60.

7. Koppelmans V, Breteler MM, Boogerd W, et al. Neuropsychological performance in survivors of breast cancer more than 20 years after adjuvant chemotherapy. Journal of Clinical Oncology. 2012;30:1080-6.

8. ReidArndt SA, Hsieh C, Perry MC. Neuropsy-chological functioning and quality of life during the first year after completing chemotherapy for breast cancer. Psychooncology. 2010;19:535-44.

9. Dubruille $\mathrm{S}$, Libert $\mathrm{Y}$, Roos $\mathrm{M}$, et al. Identification of clinical parameters predictive of 1-year survival using two geriatric tools in clinically fit older patients with hematological malignancies: major impact of cognition. Journal of Geriatric Oncology. 2015;6:362-9.

10. Abdel Rahman T, El Gaafary M. Montreal cognitive assessment Arabic version: reliability and validity prevalence of mild cognitive impairment among elderly attending geriatric clubs in Cairo. Geriatrics and gerontology international. 2009. https://doi.org/10.1111/-j.1447-05940.2008.00509.x.

11. Geissler K, Koller E, Hubmann E, Niederwieser D, Hinterberger W, Geissler D, et al. Granulocyte colony-stimulating factor as an adjunct to induction chemotherapy for adult acute lymphoblastic leukemia--a randomized phase-III study. Blood. 1997:90(2):590-6.

12. Quesnel C, Savard J, Ivers H. Cognitive impairments associated with breast cancer treatments: results from a longitudinal study. Breast Cancer Res Treat. 2009;116(1):113-23.

13. Collins B, Mackenzie J, Stewart A, Bielajew C, Verma S. Cognitive effects of chemotherapy in post-menopausal breast cancer patients 1 year after treatment. Psychooncology. 2009;18(2):134-43.

14. Ahles TA, Root JC, Ryan EL. Cancer- and cancer treatment-associated cognitive change: an update on the state of the science. Journal of Clinical Oncology. 2012;30(30):3675-86

15. Ahles TA, Saykin AJ, Furstenberg CT, Cole B, Mott LA, Skalla K, et al. Neuropsychologic impact of standard-dose systemic chemotherapy in longterm survivors of breast cancer and lymphoma. Journal of Clinical Oncology. 2002;20(2):485-93.

16. Brezden CB, Phillips KA, Abdolell $M$, et al. Cognitive function in breast cancer patients receiving adjuvant chemotherapy. J ClinOncol. 2000;18: 2695-701.

17. Von derWeid N, Mosimann I, Hirt A, et al. Intellectual outcome in children and adolescents with acute lymphoblastic leukaemia treated with chemotherapy alone: age- and sex-related differences. Eur J Cancer. 2003;39: 359-65.

18. Castellon SA, Ganz PA, Bower JE, et al. Neurocognitive performance in breast cancer survivors exposed to adjuvant chemotherapy and tamoxifen. J. Clin. Exp. Neuropsychol. 2004;26:955-69.

19. Jenkins V, Shilling V, Deutsch G, et al. A 3-year prospective study of the effects of adjuvant treatments on cognition in women with early stage breast cancer. Br J Cancer. 2006;94(6):828-34.

20. Donovan KA, Small BJ, Andrykowski MA, et al. Cognitive functioning after adjuvant chemotherapy and/or radiotherapy for early-stage breast carcinoma. Cancer. 2005;104(11):2499-507.

21. AndersonV GT, Smibert E, et al. Neurobehaviouralsequelae following cranial irradiation and chemotherapy in children: an analysis of risk factors. PediatrRehabil. 1997;1:63-76.

22. Vardy J, Wefel JS, Ahles T, et al. Cancer and cancer-therapy related cognitive dysfunction: an international perspective from the Venice cognitive workshop. Annals of Oncology. 2008;19:623-9.

23. Peckham VC. Learning disorders associated with the treatment of cancer in childhood. Journal of the Association of Pediatric Oncology Nursing. 1988;5:10-3.

24. Peckham VC. Learning disabilities in long-term survivors of childhood cancer: concerns for parents and teachers. Reading, Writing and Learning Disabilities. 1989;5:313-25.

25. Loh KP, Janelsins MC, Mohile SG et al. (2016): Chemotherapy-related cognitive impairment in older patients with cancer J Geriatr Oncol. Jul; $7(4)$ : 270-280.

26. Sorror ML, Maris MB, Storb R, Baron F, Sandmaier BM, Maloney DG, Storer B. Hematopoietic cell transplantation (HCT)-specific comorbidity index: a new tool for risk assessment before allogeneic HCT. Blood. 2005;106(8):2912-9.

27. Panza F, Frisardi V, Capurso C, Imbimbo BP, Vendemiale G, Santamato A Solfrizzi V. Metabolic syndrome and cognitive impairment: current epidemiology and possible underlying mechanisms. J Alzheimers Dis. 2010; 21(3):691-724.

28. Janelsins MC, Mustian KM, Palesh OG, Mohile SG, Peppone LJ, Sprod LK, et al.(2012): Differential expression of cytokines in breast cancer patients receiving different chemotherapies: implications for cognitive impairment research. Support Care Cancer Off J Multinatl Assoc Support Care Cancer. Apr; 20(4):831-839.

29. Buizer Al, de Sonneville LM, van den Heuvel-Eibrink MM, et al. Chemotherapy and attentional dysfunction in survivors of childhood acute lymphoblastic leukemia: Effect of treatment intensity. Pediatr Blood Cancer. 2005:45:281-90

30. Schmithorst VJ, Holland SK, Dardzinski BJ. Developmental differences in white matter architecture between boys and girls. Hum Brain Mapp. 2008; 29:696-710

31. Heaton RK, Franklin DR, Ellis RJ, McCutchan JA, Letendre SL, LeBlanc S. ... for the CHARTER and HNRC Groups. (2011). HIV-associated neurocognitive disorders before and during the era of combination antiretroviral therapy: differences in rates, nature, and predictors. Journal of Neurovirology. 17(1):3-16.

32. Meyers CA, Albitar M, Estey E. Cognitive impairment, fatigue, and cytokine levels in patients with acute myelogenous leukemia or myelodysplastic syndrome. Cancer. 2005;104:788-93.

33. Meadows ME, Chang G, Jones JA, Antin JR, Orav EJ. Predictors of neuropsychological change in patients with chronic myelogenous leukemia and myelodysplastic syndrome. Arch Clin Neuropsychol. 2013;28:363-74.

34. Jones D, Vichaya EG, Wang XS, Sailors MH, Cleeland CS, Wefel JS. Acute cognitive impairment in patients with multiple myeloma undergoing autologous hematopoietic stem cell transplant. Cancer. 2013;119:4188-95.

35. Cheung YT, Lim SR, Ho HK, Chan A. Cytokines as mediators of chemotherapy-associated cognitive changes: current evidence, limitations and directions for future research. PLoS One. 2013;8(12):e81234.

\section{Publisher's Note}

Springer Nature remains neutral with regard to jurisdictional claims in published maps and institutional affiliations.

\section{Submit your manuscript to a SpringerOpen ${ }^{\circ}$ journal and benefit from:}

- Convenient online submission

- Rigorous peer review

- Open access: articles freely available online

High visibility within the field

- Retaining the copyright to your article

Submit your next manuscript at $\boldsymbol{\nabla}$ springeropen.com 